\title{
Changing hearts and minds
}

\author{
Elizabeth Loder head of research
}

The BMJ

The UK is no longer measles free, and other vaccine preventable childhood illnesses are on the rise. What is the best way to counter the claims of the antivaccination movement? Not with papers, peer reviewed studies, or symposiums, say Kathryn Perera and colleagues (doi:10.1136/bmj.16447). "Don’t bring a fact to a narrative fight," they advise. The "we know best" approach is not effective in a world that is sceptical of the "old power" represented by medical experts and institutions. Instead, those who hope to change minds must embrace "new power" approaches that take into account the emotional explanations for beliefs and attitudes. The success of the antivaccination lobby is "based on tactics and thinking that can and should be deployed against it." These strategies rely on stories and context that are created and shared in the peer to peer communities that thrive on social media platforms.

Meanwhile, the Daily Mail has launched a campaign to "end the MMR vaccine ignorance" and encourage parents to inoculate their children. The newspaper expresses "profound regret" for the credence it gave to the now retracted paper by Andrew Wakefield and colleagues linking the MMR vaccine to autism. But is the Mail's apology too late? Ingrid Torjesen examines charges of hypocrisy levelled at the paper (doi:10.1136/bmj. 16487). It is widely viewed as having contributed to vaccine hesitancy with articles that were heavily slanted towards antivaccination arguments. A new editor has come in and taken a fresh look, and this as much as the resurgence of disease may have contributed to the Mail's change of heart.

How easy will it be to change minds about the use of platelet rich plasma to treat acute ruptures of the Achilles tendon? These injections are widely used for a variety of musculoskeletal and even cosmetic complaints, but convincing evidence of benefit is lacking for most indications. David Keene and colleagues randomised 230 adults with acute rupture of the Achilles tendon to have an injection of platelet rich plasma or placebo dry needling (doi:10.1136/bmj.16132). Both groups received standard rehabilitative care. At 24 weeks there was no difference between the groups in muscle tendon function or on other measured outcomes.

Mark Skopec and colleagues suggest that we should change our view of healthcare innovations and recognise that rich countries can benefit from adopting technologies developed in low resource settings (doi:10.1136/bmj.16205). This sort of "reverse innovation" upends the idea that progress and improvements in healthcare naturally flow from high to low income settings. Examples abound, including the non-surgical Ponseti technique for treating talipes equinovarus (clubfoot). Developed in African countries with limited access to expensive surgical interventions, this is now the recommended treatment in most high income countries too. 